\title{
Glycoconjugates (Glycosaminoglycans and Glycoproteins) and Glycogen in the Human Cervix Uteri
}

\author{
Takeo Shimizu, ${ }^{*}$ Masahiko Endo $\dagger$ and Zensaku \\ Yosizawa \\ Department of Biochemistry, Tohoku University School of \\ Medicine, Sendai 980
}

\begin{abstract}
Shimizu, T., Endo, M. and Yosizawa, Z. Glycoconjugates /Glycosaminoglycans and Glycoproteins) and Glycogen in the Human Cervix Uteri. Tohoku J. exp. Med., 1980, 131 (3), 289-299 — Glycoconjugates and glycogen obtained from the cervix uteri of nonpregnant women of proliferative phase and secretory phase, and from the postpartum cervix uteri were determined by electrophoresis on cellulose acetate membrane and chemical analysis before and after enzymatic digestion and nitrous acid treatment. Although the contents of neutral glycopeptide fraction in these tissues were similar, the glycogen content in the postpartum tissue greatly decreased. The contents in terms of hexosamine of hyaluronic acid, chondroitin sulfate, heparan sulfate and acidic glycopeptides in the postpartum tissue greatly increased in comparison with those in the nonpregnant tissues, whereas that of dermatan sulfate slightly decreased. In addition, dermatan sulfate in the postpartum tissue contained a small portion of non-sulfated disaccharide units, which was not detected in the nonpregnant tissues. The results indicate that significant changes in the contents of glycoconjugates and glycogen of human cervix uteri occur in ripening. The roles of these substances in the cervical ripening are discussed.—- glycoconjugate; glycosaminoglycan; glycoprotein; glycogen; cervix uteri; cervical ripening
\end{abstract}

Although glycoconjugates (GC) [glycosaminoglycans (GAG) and glycoproteins (GP)] of the human cervix uteri have been studied by many investigators (Buckingham et al. 1962; Dickey et al. 1966; Stevens et al. 1966; Zachariae and Thorsøe 1966; Bryant et al. 1968; Endo 1969; Ohkawa 1969; Kure 1970; Majima 1971; André et al. 1972; Kondo 1972; Gröschel-Stewart et al. 1973; Nakaya 1973; Danforth et al. 1974; Karube et al. 1975) concerning with ripening, the precise roles of $\mathrm{GC}$ in the changes which occur in the human cervix uteri prior to the onset of labor remained to be elucidated.

Received for publication, July 7, 1979.

* Present address: Department of Obstetrics and Gynecology, Tohoku University School of Medicine, Sendai 980.

$\dagger$ Present address: Department of Biochemistry, Hirosaki University School of Medicine, Hirosaki 036.

Abbreviations: GC, glycoconjugates; GAG, glycosaminoglycans; GP, glycopeptides; $\triangle \mathrm{Di}$-0S, 2-acetamido-2-deoxy-3-0-(4-deoxy-a-L-threo-hex-4-enopyranosyluronic acid)-D-ga lactose; $\triangle \mathrm{Di}-4 \mathrm{~S}, \Delta \mathrm{Di}-6 \mathrm{~S}$ and $\Delta \mathrm{Di}$-diS, 4 -sulfate, 6 -sulfate and disulfate, respectively, of $\Delta \mathrm{Di}$ OS; CPC, cetylpyridinium chloride. 
In order to elucidate the role of each GC in ripening, we studied on GAG, GP and glycogen in the human cervix uteri of nonpregnant women of proliferative phase and secretory phase and in the postpartum cervix uteri. This paper reports the results of the study.

\section{Materials and Methods}

Materials. Streptomyces hyaluronidase, chondroitinase AC-II, chondroitinase $\mathrm{ABC}$, pronase $\mathrm{P}, \boldsymbol{a}$-amylase, cellulose acetate membrane (Separax), Alcian blue 8GS, toluidine blue, $\alpha_{1}$-acid glycoprotein and standard GAG were the same as reported previously (Endo et al. 1978, 1980; Endo and Yosizawa 1979). $\Delta \mathrm{Di}-4 \mathrm{~S}, \Delta \mathrm{Di}-6 \mathrm{~S}, \Delta \mathrm{Di}$-diS and $\Delta \mathrm{Di}-0 \mathrm{~S}$ were obtained from Seikagaku Kogyo Co., Tokyo. Other materials were commercial products.

Cervix uteri. The nonpregnant cervices uteri of proliferative phase and secretory phase were obtained from the uteri operatively removed from leiomyoma of premenopausal Japanese women. The postpartum cervices uteri were obtained from the uteri operatively removed from cervical laceration and for atonic hemolysis. Approximately $10 \mathrm{~g}$ (wet weight) of the part of the cervix between the internal and external os were excised from each uterus. The tissues certified as normal by histological examination were used as the starting material.

Separation of crude GC. Each cervix tissue was cut into small pieces, and homogenized with $30 \mathrm{ml}$ of ethanol $(80 \%)$ with a Waring Blender. The homogenate was then heated for $3 \mathrm{~min}$ at $100^{\circ} \mathrm{C}$. The suspension was cooled in ice-water, and centrifuged. The sediment was washed twice with ethanol, and once with ether, then dried over $\mathrm{CaCl}_{2}$ in vacuo. Crude $\mathrm{GC}$ were obtained by repeated digestion of the above powder $(2 \mathrm{~g})$ with pronase $\mathrm{P}$ (80 mg in total) at $\mathrm{pH} 8.0$ as described previously (Endo et al. 1978). To promote proteolysis, however, the solution of the first pronase digest was heated for $5 \mathrm{~min}$ at $100^{\circ} \mathrm{C}$ prior to second pronase digestion.

Fractionation of crude GC with CPC. The above crude GC were fractionated with CPC as described previously (Endo et al. 1978). The substances recovered from the CPCprecipitate as described previously (Endo et al. 1978) were dissolved in a small volume of water. The solution was dialyzed in a Visking tubing against several changes of distilled water for 2 days. The non-dialyzable fraction was filtered through a glass microfiber filter (Whatmann), and the filtrate was concentrated, then lyophilized, yielding CPC-ppt Fr. On the other hand, 4 volumes of ethanol saturated with $\mathrm{NaCl}$ were added to the CPCsupernatant solution. The resulting precipitate was collected by centrifugation, then dissolved in a small volume of water, followed by the same treatments as above, yielding CPC-sup Fr.

Quantitation of glycogen and neutral GP Fr in CPC-sup Fr. a-Amylase $(1 \mathrm{mg})$ and a small amount of toluene were added to a solution $(1 \%)$ of the above CPC-sup Fr in $0.01 \mathrm{M}$ phosphate buffer $(\mathrm{pH} 6.0)$ containing $0.15 \mathrm{M} \mathrm{NaCl}$. The mixture was then incubated for $12 \mathrm{hr}$ at $37^{\circ} \mathrm{C}$. An aqueous trichloroacetic acid $(50 \%)$ was added to the incubation mixture at $4^{\circ} \mathrm{C}$ to give a final concentration of $7 \%$, and the resulting precipitate was removed by centrifugation. Four volumes of ethanol saturated with $\mathrm{NaCl}$ were added to the supernatant, and the mixture was left to stand for several hours at $4^{\circ} \mathrm{C}$. The precipitate thus formed was collected by centrifugation, then dissolved in a small volume of water. The solution was dialyzed in a Visking tubing against distilled water. The non-dialyzable fraction was concentrated, then lyophilized, yielding neutral GP Fr. The content of glycogen was calculated by subtraction of the amount of neutral GP Fr from that of CPC-sup Fr.

Quantitation of hyaluronic acid, chondroitin sulfate and dermatan sulfate in CPC-ppt Fr by digestion with mucopolysaccharidases. Determination of GAG by digestion with mucopolysaccharidases was carried out by a modification of the method of Endo et al. (1978). An aliquot $(100 \mu l)$ of an aqueous solution $(0.5 \%)$ of CPC-ppt Fr was added with a half volume of the enzyme solution of Streptomyces hyaluronidase or chondroitinase AC-II or chondroitinase $\mathrm{ABC}$ described in a previous paper (Endo et al. 1978). Water was used 
instead of enzyme solution for control experiment. After the incubation, 4 volumes of ethanol saturated with $\mathrm{NaCl}$ were added to the incubation mixture, and the mixture was cooled in ice-water. The precipitate was collected by centrifugation, washed with 300 $\mu \mathrm{l}$ of ethanol. The supernatant and the washing were combined, and the solution was evaporated in vacuo to dryness. The residue was then hydrolyzed with $4 \mathrm{M} \mathrm{HCl} \mathrm{for} 8 \mathrm{hr}$ at $100^{\circ} \mathrm{C}$, and the content of hexosamine in the hydrolyzate was determined. The contents of hexosamine in the ethanol-soluble fractions obtained by digestions with Streptomyces hyaluronidase, chondroitinase AC-II and chondroitinase ABC were expressed as $[\mathrm{A}],[\mathrm{B}]$ and $[\mathrm{C}]$, respectively. The contents in terms of hexosamine of hyaluronic acid, chondroitin sulfate and dermatan sulfate can be calculated as follows: hyaluronic acid $=[\mathrm{A}]$; chondroitin sulfate $=[B]-[A]$; dermatan sulfate $=[C]-[B]$. The substances remained after digestion with these enzymes were examined by electrophoresis on cellulose acetate membrane.

Quantitation of heparan sulfate and acidic GP in CPC.ppt Fr by nitrous acid treatment. An aliquot $(100 \mu \mathrm{l})$ of the above CPC-ppt Fr was digested with chondroitinase ABC as above. The sediment precipitated by the addition of 4 volumes of ethanol saturated with $\mathrm{NaCl}$ was dissolved in water $(0.2 \mathrm{ml})$. A half of this solution was evaporated to dryness, and the residue was hydrolyzed with $4 \mathrm{M} \mathrm{HCl}$ for $8 \mathrm{hr}$ at $100^{\circ} \mathrm{C}$. The content of hexosamine in the hydrolyzate was determined, and expressed as [D]. To the remaining solution $(0.1 \mathrm{ml})$ were added $0.1 \mathrm{ml}$ of an aqueous sodium nitrite $(5 \%)$ and $0.1 \mathrm{ml}$ of acetic acid $(33 \%)$ with stirring. The mixture was left to stand for $1 \mathrm{hr}$ at room temperature, followed by addition of $1.2 \mathrm{ml}$ of ethanol saturated with $\mathrm{NaCl}$. The precipitate was collected by centrifugation, washed with ethanol. A half of the precipitate was then hydrolyzed with $4 \mathrm{M} \mathrm{HCl}$ for $8 \mathrm{hr}$ at $100^{\circ} \mathrm{C}$. The content of hexosamine in the hydrolyzate was determined, and expressed as [E]. The substances remained after the nitrous acid treatment were examined by electrophoresis on cellulose acetate membrane. Since no heparin nor keratan sulfate was detected in CPC-ppt $\mathrm{Fr}$ by electrophoresis to be described later, the contents in terms of hexosamine of heparan sulfate and acidic GP can be calculated as follows: heparan sulfate $=[\mathrm{D}] \times 2-[\mathrm{E}] \times 4$; acidic $\mathrm{GP}=[\mathrm{E}] \times 4$.

Determination of unsaturated disaccharides in the digests with chondroitinase AC.II and chondroitinase ABC. CPC-ppt Fr was digested with Streptomyces hyaluronidase as above. The substances resistant to this enzyme were then digested with chondroitinase AC-II as above. The substances remained after chondroitinase AC digestion were further digested with chondroitinase $A B C$ as above. Unsaturated disaccharides in the digests with chondroitinase AC-II and chondroitinase $\mathrm{ABC}$ were determined by the method of Suzuki (1960).

Electrophoresis. Electrophoresis on cellulose acetate membrane was carried out as described previously (Endo and Yosizawa 1975).

Determination of constituents. Monosaccharides in an acid hydrolyzate $(1 \mathrm{M} \mathrm{HCl}$, $8 \mathrm{hr}, 100^{\circ} \mathrm{C}$ ) of neutral GP Fr were qualitatively determined by paper chromatography. Paper chromatography was carried out on Whatman No. 1 filter paper $(12 \times 60 \mathrm{~cm})$, developed with $n$-butanol-pyridine-water $(6: 4: 3$, by volume). by a descending technique for $18 \mathrm{hr}$ at $22^{\circ} \mathrm{C}$. The substances on the paper were located by staining with alkaline silver reagent. Hexosamine in an acid hydrolyzate $\left(4 \mathrm{M} \mathrm{HCl}, 8 \mathrm{hr}, 100^{\circ} \mathrm{C}\right)$ was determined by the method of Blix (1948).

\section{Results}

\section{Water content of cervix uteri}

The nonpregnant cervix uteri of proliferative phase and secretory phase, and the postpartum cervix uteri were treated with boiling ethanol, and then dried. The water content in the postpartum tissue was slightly higher than those in the nonpregnant tissues (Table 1). 
TaBLE 1. Water contents and yields of crude $G C$

\begin{tabular}{cccc} 
Tissue & $\mathrm{N}^{*}$ & $\begin{array}{c}\text { Water content } \\
\text { (mg/g of wet tissue) }\end{array}$ & $\begin{array}{c}\text { Yield of GC } \\
\text { (mg/g of dry tissue) }\end{array}$ \\
\hline $\begin{array}{c}\text { Nonpregnant } \\
\text { Proliferative phase }\end{array}$ & 14 & $781 \pm 31 \dagger$ & $29.02 \pm 2.89 \dagger$ \\
Secretory phase & 12 & $797 \pm 29$ & $29.10 \pm 2.74$ \\
Postpartum & 19 & $836 \pm 25$ & $37.93 \pm 4.98$ \\
\hline
\end{tabular}

* Number of cervix uteri examined.

$\uparrow$ Standard deviation.

\section{Separation of crude $G C$}

The dried tissues were subjected to repeated pronase digestion. Yields of the resulting crude GC are shown in Table 1. The yields (mg/g of dry tissue) of crude GC from the nonpregnant tissues of proliferative phase and secretory phase were similar, whereas that from the postpartum tissue was higher than those from the nonpregnant tissues.

\section{Fractionation of crude $G C$ with $C P C$}

Fractionation of crude GC with CPC gave CPC-ppt Fr and CPC-sup Fr. Recoveries of these fractions are shown in Table 2. The ratios of CPC-ppt Fr to CPC-sup Fr from the nonpregnant samples were substantially identical, and were approximately $39: 61$, while inverse ratio was observed on these fractions from the postpartum sample.

TABLE 2. Recoveries* of CPC-sup Fr and CPC-ppt Fr

\begin{tabular}{lrr}
\hline Tissue & CPC-sup Fr & CPC-ppt Fr \\
\hline Nonpregnant & & \\
Proliferative phase & $14.71 \pm 2.65+(61.0)$ & $9.36 \pm 1.56 \dagger(39.0)$ \\
Secretory phase & $14.15 \pm 1.86(61.2)$ & $8.90 \pm 2.03(38.8)$ \\
Postpartum & $9.83 \pm 2.10(38.3)$ & $15.64 \pm 4.37(61.8)$ \\
\hline * Expressed as mg/g of dry tissue (data in parentheses are \\
percent recoveries in total). Numbers of cervices uteri examined \\
were the same as in Table 1. \\
† Standard deviation.
\end{tabular}

\section{Glycogen and neutral GP Fr in CPC-sup Fr}

Electrophoretograms of CPC-sup Fr on cellulose acetate membrane are shown in Fig. 1. All the samples examined gave three major bands stainable by the PASreaction. Of these bands, the slowest moving bands corresponded to that of glycogen, and disappeared after $\alpha$-amylase digestion, indicating that they contained glycogen. The fastest moving bands corresponded to that of $\alpha_{1}$-acid glycoprotein, so they might contain sialo GP. The constituent monosaccharides in the acid hydrolyzate $\left(1 \mathrm{M} \mathrm{HCl}, 8 \mathrm{hr}, 100^{\circ} \mathrm{C}\right)$ of the substances remained after $\alpha$-amylase digestion were examined by paper chromatography. The results showed that all 


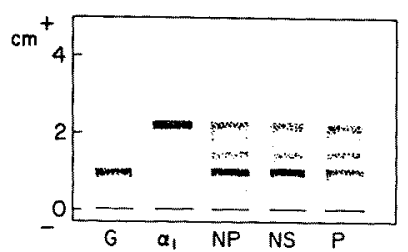

Fig. 1. Tracing of electrophoretograms of CPC-sup $\mathrm{Fr}$ obtained from the nonpregnant cervix uteri of proliferative phase (NP) and secretory phase (NS) and from the postpartum cervix uteri (P). Electrophoresis was carried out on cellulose acetate membrane (Separax) in formic acid-pyridine buffer $(\mathrm{pH} 3.0$ ) at $1 \mathrm{~mA} / \mathrm{cm}$ for $40 \mathrm{~min}$, stained by the PAS reaction. $G$, glycogen; $\alpha_{1}, \alpha_{1}$-acid glycoprotein.

the samples examined contained galactose and glucosamine as the major sugars, together with mannose, glucose, galactosamine and L-fucose as the minor ones. No difference was observed on their constituent monosaccharides among these samples. These observations suggest that the substances remained after $\alpha$ amylase digestion are regular neutral GP and sialo GP.

The content of glycogen in CPC-sup Fr was determined by $\alpha$-amylase digestion, and that of neutral GP Fr was calculated as described in Materials and Methods. The data are shown in Table 3. The content ( $\mathrm{mg} / \mathrm{g}$ of dry tissue) of glycogen in the nonpregnant tissue of proliferative phase was slightly higher than that of secretory phase, whereas that of the postpartum tissue was significantly lower than those of the nonpregnant tissues. On the other hand, the contents (mg/g of dry tissue) of neutral GP Fr were similar in these three tissues. These observations suggest that consumption of glycogen occur in ripening of the cervix uteri without any change in neutral GP Fr in CPC-sup Fr.

TABLE 3. Contents* of glycogen and neutral GP Fr in CPC-Sup Fr

\begin{tabular}{ccc} 
Tissue & Glycogen & Neutral GP Fr \\
\hline Nonpregnant & & \\
Proliferative phase & $7.34(49.3)$ & $7.37(50.7)$ \\
Secretory phase & $6.43(44.3)$ & $7.73(55.7)$ \\
Postpartum & $2.77(27.8)$ & $7.00(72.2)$ \\
\hline
\end{tabular}

* Expressed as $\mathrm{mg} / \mathrm{g}$ of dry tissue (data in parentheses are percent contents in total). Data are mean values of 3 experiments, in which substantially identical values were obtained.

\section{GAG and acidic GP in CPC-ppt Fr}

Electrophoretograms of CPC-ppt $\mathrm{Fr}$ before and after digestions with Streptomyces hyaluronidase, chondroitinase $\mathrm{AC}-\mathrm{II}$ and chondroitinase $\mathrm{ABC}$ are shown in Fig. 2. Similar figures were observed on the samples from the nonpregnant tissues of proliferative phase and secretory phase. However, electrophoretograms of the postpartum samples differed from those of the nonpregnant samples in the intensity of the bands. The bands corresponded to that of hyaluronic acid were 
not detected after digestion with Streptomyces hyaluronidase, indicating that they contained hyaluronic acid. Since the fastest moving bands disappeared after digestion with chondroitinase $\mathrm{AC}$, they might contain chondroitin sulfate. Most of the substances in the bands corresponding to dermatan sulfate were susceptible to chondroitinase $\mathrm{ABC}$, so they might be dermatan sulfate.

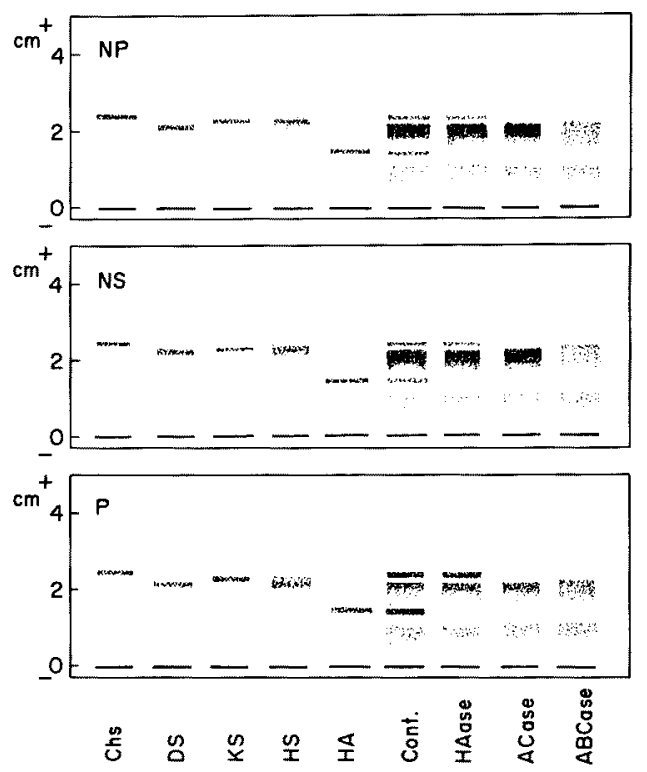

Fig. 2. Tracing of electrophoretograms of CPC-ppt Fr before (Cont.) and after digestions with Streptomyces hyaluronidase (HAase), chondroitinase AC-II (ACase) and chondroitinase $A B C$ (ABCase) of the nonpregnant cervix uteri of proliferative phase (NP) and secretory phase (NS) and of the postpartum cervix uteri (P). Electrophoresis was carried out on Separax in formic acid-pyridine buffer (pH 3.0 ) at $1 \mathrm{~mA} / \mathrm{cm}$ for 20 min, stained with Alcian blue-ethanol $(0.05 \%$ in $70 \%$ ethanol). Chs, chondroitin sulfate $A$ and/or C; DS, dermatan sulfate; KS, keratan sulfate; HS, heparan sulfate; HA, hyaluronic acid.

The fastest moving bands remained after chondroitinase $\mathrm{ABC}$ digestion might contain heparan sulfate, since the intensity of the color stained with Alcian blue-HCl $(0.05 \%$ in $0.1 \mathrm{M} \mathrm{HCl})$ reduced after nitrous acid treatment. The mobility of these bands was lower than that of authentic heparan sulfate, indicating that heparan sulfate in the cervix uteri might contain less sulfates than authentic one. A certain portion of the above bands still remained after nitrous acid treatment, and were stainable by the $\mathrm{PAS}$ reaction, as well as with Alcian blue- $\mathrm{HCl}$, suggesting the presence of sulfated GP in these bands (Endo and Yosizawa 1979). The slower moving bands remained after nitrous acid treatment were stainable by the PAS reaction and with Alcian blue-ethanol $(0.05 \%$ in $70 \%$ ethanol), but negative for Alcian blue- $\mathrm{HCl}$ staining, suggesting that these bands might contain $\mathrm{CPC}$ precipitable sialo GP. 
The nonpregnant tissues of proliferative phase contained slightly more hyaluronic acid, chondroitin sulfate and acidic GP than those of secretory phase, whereas the opposite was the cases of dermatan sulfate and heparan sulfate (Table 4). Dermatan sulfate was predominant in these tissues. The differences in the contents ( $\mathrm{mg} / \mathrm{g}$ of dry tissue) in terms of hexosamine of GAG and acidic GP between the nonpregnant tissues and the postpartum tissue were remarkable. The contents of hyaluronic acid, chondroitin sulfate, heparan sulfate, and acidic GP in the postpartum tissue greatly increased in comparison with those in the nonpregnant tissues, whereas that of dermatan sulfate slightly decreased.

TABLE 4. Contents* of GAG and acidic GP in CPC.ppt Fr

\begin{tabular}{|c|c|c|c|c|c|c|}
\hline \multirow{2}{*}{ Tissue } & \multirow{2}{*}{$\begin{array}{l}\text { Hyaluronic } \\
\text { acid }\end{array}$} & \multicolumn{2}{|c|}{ Chondroitin sulfate } & \multirow{2}{*}{$\begin{array}{l}\text { Dermatan } \\
\text { sulfate }\end{array}$} & \multirow{2}{*}{$\begin{array}{l}\text { Heparan } \\
\text { sulfate }\end{array}$} & \multirow{2}{*}{$\begin{array}{l}\text { Acidic } \\
\text { GP }\end{array}$} \\
\hline & & Total & $\begin{array}{l}\Delta \mathrm{Di}-4 \mathrm{~S} / \\
\Delta \mathrm{Di}-6 \mathrm{~S} \dagger\end{array}$ & & & \\
\hline \multicolumn{7}{|l|}{ Nonpregnant } \\
\hline Proliferative phase & $1.09(11.6)$ & $0.87(9.3)$ & $87.0 / 13.0$ & $6.18(66.0)$ & $0.45(4.8)$ & $0.77(8.2)$ \\
\hline Secretory phase & $0.90(10.1)$ & $0.62(7.0)$ & $89.7 / 10.3$ & $6.29(70.5)$ & $0.52(5.8)$ & $0.59(6.6)$ \\
\hline Postpartum & $2.72(17.7)$ & $2.34(15.2)$ & $88.8 / 11.2$ & $5.18(33.7)$ & 2. $18(14,2)$ & $2.95(19.2)$ \\
\hline
\end{tabular}

* Expressed as mg (in terms of hexosamine)/g of dry tissue (data in parentheses are percent contents in total). Data are mean values of 3 experiments, in which substantially identical values were obtained.

$\dagger$ Percent ratio of unsaturated disaccharides in the digests with chondroitinase AC-II.

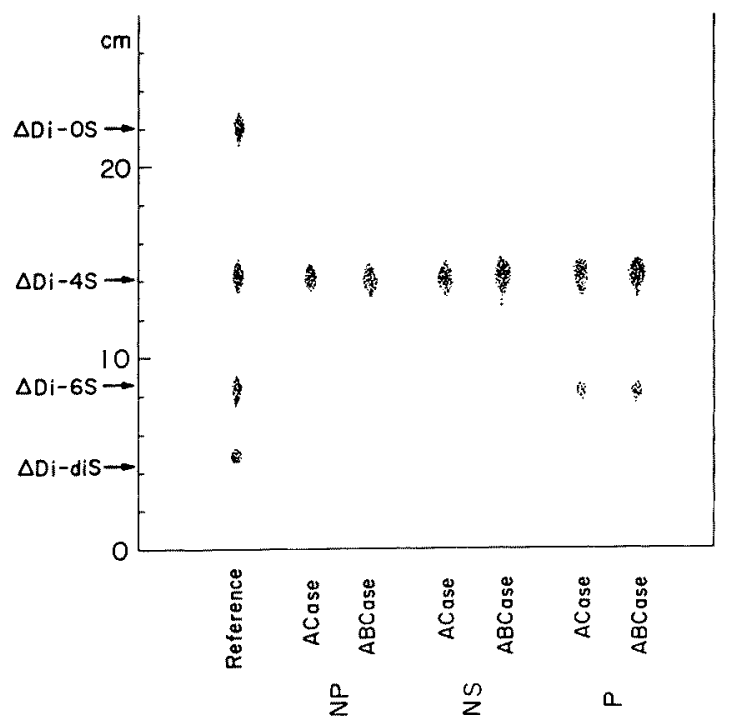

Fig. 3. Tracing of paper chromatograms of unsaturated disaccharides in the digests of CPC-ppt Fr with chondroitinase AC-II (ACase) and chondroitinase ABC (ABCase) of the nonpregnant cervix uteri of proliferative phase (NP) and secretory phase (NS) and the postpartum cervix uteri $(\mathrm{P})$, stained with alkaline-silver reagent. Paper chromatography was carried out as described in the text. 
To determine the ratio of chondroitin 4-sulfate and chondroitin 6-sulfate in chondroitin sulfate, the substances resistant to Streptomyces hyaluronidase were digested with chondroitinase AC-II, and then unsaturated disaccharides in the digests were examined. The results (Fig. 3 and Table 4) showed that more than $87 \%$ of unsaturated disaccharides was $\triangle \mathrm{Di}-4 \mathrm{~S}$ and the rest was $\triangle \mathrm{Di}-6 \mathrm{~S}$, indicating that chondroitin 4-sulfate was predominant in the cervix uteri with a small portion of chondroitin 6-sulfate. Since neither $4 \mathrm{Di}$-0S nor $\triangle \mathrm{Di}$-diS was detected in the digests, non-sulfated and over-sulfated disaccharide units might not present in the polysaccharide chains of chondroitin sulfate.

On the other hand, the digests with chondroitinase $\mathrm{ABC}$ of dermatan sulfate from the above three tissues contained $4 \mathrm{Di}-4 \mathrm{~S}$ as the major unsaturated disaccharides and $4 \mathrm{Di}-6 \mathrm{~S}$ as the minor ones (Fig. 3). This finding indicates that dermatan sulfate in the cervix uteri has hybrid polysaccharide chains. In addition, dermatan sulfate in the postpartum tissue might contain a small portion of non-sulfated disaccharide units in the polysaccharide chains, since the digests with chondroitinase $\mathrm{ABC}$ contained a small amount of $\triangle \mathrm{Di}-0 \mathrm{~S}$.

\section{Discussion}

The striking physical and chemical changes that occur in the cervix uteri in the pregnancy and labor must be involved in remodeling of the matrix in connective tissue. Since the remodeling may be caused by changes in the metabolism of $\mathrm{GC}$, collagen and related substances, there have been many investigations concerning cervical GAG and GP (Buckingham et al. 1962; Dickey et al. 1966; Stevens et al. 1966; Zachariae and Thorsøe 1966; Bryant et al. 1968; Endo 1969; Ohkawa 1969; Kure 1970; Majima 1971; André et al. 1972; Kondo 1972; GröschelStewart et al. 1973; Nakaya 1973; Danforth et al. 1974; Karube et al. 1975). However, the results thus far obtained are insufficient to explain the dramatic changes of the cervix uteri in ripening.

In order to elucidate the precise remodeling figures of GC in the cervix uteri in ripening, we studied GAG and GP in the nonpregnant tissues of proliferative phase and secretory phase and in the postpartum tissue.

The content of water in the postpartum tissue was slightly higher than those in the nonpregnant tissues (Table 1). This observation was consistent with those reported by Bryant et al. (1968) and Karube et al. (1974). Since the cervix uteri was elastic hard, proteolysis was repeated after heat denaturation of protein remained after first pronase digest. By this treatment, the yields of crude GC greatly increased compared to those reported previously (Endo 1969; Ohkawa 1969; Majima 1971; Nakaya 1973). Although no difference was observed between the yields ( $\mathrm{mg} / \mathrm{g}$ of dry tissue) of crude GC from the nonpregnant tissues of proliferative phase and secretory phase, the postpartum tissue contained much more crude GC than the nonpregnant tissues (Table 1). Among crude GC, the recovery (mg/g of dry tissue) of CPC-sup Fr from the postpartum tissue was smaller than those from 
the nonpregnant tissues, while inverse relationship was observed on those of CPCppt Fr (Table 2).

To determine the contents of glycogen, neutral GP Fr, GAG and acidic GP, enzymatic digestion and nitrous acid treatment were performed. The constituent monosaccharides and the contents ( $\mathrm{mg} / \mathrm{g}$ of dry tissue) of neutral GP Fr in CPCsup Fr were similar in the above three tissues, but the glycogen content in CPCsup Fr of the postpartum tissue greatly decreased. These observations suggest that neutral GP and sialo GP in the CPC-sup Fr do not play an important role in ripening, whereas glycogen may supply energy. Change of the glycogen content of ectocervical tissue of females receiving steroid was already reported (Gregoire and Ledger 1969).

Although slight changes in the contents (mg/g of dry tissue) in terms of hexosamine of each GAG in the nonpregnant tissues between proliferative phase and secretory phase were observed, those of hyaluronic acid, chondroitin sulfate, heparan sulfate and acidic GP in the postpartum tissue greatly increased in comparison with those in the nonpregnant tissues, but that of dermatan sulfate in the former slightly decreased. Similar results were reported by Nakaya (1973), except for heparan sulfate. No datum has been reported on cervical heparan sulfate yet.

In chondroitin sulfate, chondroitin 4-sulfate was predominant with a small portion of chondroitin 6 -sulfate, although a hybrid structure of chondroitin sulfate A containing a small portion of 6 -sulfated disaccharide units could not be excluded. The ratios of chondroitin 4-sulfate to chondroitin 6-sulfate in these three tissues were similar. Contrary to this finding, Nakaya (1973) stated that the content of chondroitin 4-sulfate was less than that of chondroitin 6-sulfate in cervix uteri. Dermatan sulfate was found to be a hybrid GAG, consisting of the major 4-sulfated disaccharide units with the minor 6-sulfated disaccharide units. In addition, a small portion of non-sulfated disaccharide units was present in the polysaccharide chains of this GAG in the postpartum tissue. Decrease in the dermatan sulfate content and increase in the other GC contents in cervix uteri may be important in ripening, since dermatan sulfate is thought to be important to maintain the elastic hard nature of this tissue. Increase in the GAG contents in ripening of cervix uteri has been reported by many investigators (Buckingham et al. 1962; Bryant et al. 1968; Endo 1969; Ohkawa 1969; Kure 1970; Majima 1971; Kondo 1972; Nakaya 1973; Danforth et al. 1974), and it has been assumed that these GAG might play important roles in ripening.

Although Danforth et al. (1974) found a keratan sulfate-like substance "new component" in the dilated cervix, we could not detect it, but found sulfated GP and sialo GP. Since the contents ( $\mathrm{mg} / \mathrm{g}$ of dry tissue) in terms of hexosamine of acidic GP in CPC-ppt Fr increased in the postpartum tissue, these GP might play an important role in ripening. The presence of sulfated GP in bovine cervix was already reported by André et al. (1972). Also, Karube et al. (1975) reported increase of "structural GP" during dilatation of human cervix in pregnancy at 
term, and Gröschel-Stewart et al. (1973) reported the presence of "groundsubs" tance" in human cervix uteri. These compounds are thought to be acidic GP in the present study.

\section{Acknowledgment}

We thank Professor M. Suzuki, Department of Obstetrics and Gynecology, for sending T.S. to the Department of Biochemistry and for his interest throughout this work.

\section{References}

1) André, F., André, C., Bhushana Rao, K.S.P., Masson, P.L. \& Heremans, J.F. (1972) Use of gas-liquid chromatography in the analysis of hydrolysates of bovine-oestrus cervical glycoprotein. Carbohyd. Res., 25, 395-401.

2) Blix, G. (1948) The determination of hexosamines according to Elson and Morgan. Acta chem. scand., 2, 467-473.

3) Bryant, W.M., Greenwell, J.E. \& Weeks, P.M. (1968) Alterations in collagen organization during dilatation of the cervix uteri. Surg. Gynec. Obstet., 126, 27-39.

4) Buckingham, J.C., Selden, R. \& Danforth, D.N. (1962) Connective tissue changes in the cervix during pregnancy and labor. Ann. N.Y. Acad. Sci., 97, 733-742.

5) Danforth, D.N., Veis, A., Breen, M., Weinstein, H.G., Buckingham, J.C. \& Manalo, P. (1974) The effect of pregnancy and labor on the human cervix: Changes in collagen, glycoprotein, and glycosaminoglycans. Amer. J. Obstet. Gynec., 120, 641-651.

6) Dickey, R.P., Stevens, V.C., Vorys, N., Denko, C.W. \& Ullery, J.C. (1966) Rate of chondroitin sulfate synthesis in the cervix during pregnancy. Amer. J. Obstet. Gynec., $95,40-45$.

7) Endo, F. (1969) Study on mucopolysaccharides of the human cervix uteri Sanfujinkano-Sekai, 21, 375-381. Japanese

8) Endo, M. \& Yosizawa, Z. (1975) Glycosaminoglycans and acidic glycoproteins in rabbit uterus under estrogenic conditions. Biochim. biophys. Acta (Amst.), 404, 274-280.

9) Endo, M. \& Yosizawa, Z. (1979) A method for micro-scale isolation of sulfated glycopeptides from tissue. Tohoku J. exp. Med., 129, 225-231.

10) Endo, M., Shimizu, T., Munakata, H. \& Yosizawa, Z. (1978) Composition of acidic glycoconjugates (glycosaminoglycans and glycoprotein) in myometrium of rabbit uterus under estrogenic condition. Tohoku J. exp. Med., 126, 103-110.

11) Endo, M., Namiki, O. \& Yosizawa, Z. (1980) Fractionation with ethanol of human urinary glycosaminoglycans. Tohoku J. exp. Med., 131, 23-28.

12) Gregoire, A.T. \& Ledger, W.J. (1969) The glycogen content of human ectocervical tissue of females receiving steroid contraceptive therapy. Fertil. Steril., 20, 91-97.

13) Gröschel-Stewart, U., Hermann, U. \& Schwalm, H. (1973) "Groundsubstance" resembling amyloid extracted from the cervical portion of human cervix uteri. Brit. J. exp. Path., 54, 192-197.

14) Karube, H., Kanke, Y., Mori, Y., Hirakawa, S. \& Hayashi, M. (1975) Increase of structural glycoprotein during dilatation of human cervix in pregnancy at term. Endocr. Jap., 22, 445-448.

15) Kondo, T. (1972) Histochemical studies on the human cervix uteri. Nogoya med. $J ., 17,103-121$.

16) Kure, H. (1970) Study on ripening of the human cervix uteri. Nippon SankaFujinka Gakkai Zasshi, 22, 1227-1234. (Japanese)

17) Majima, K. (1971) Study on ripening factor of the human cervix uteri, specifically on mucopolysaccharides. Nippon Sanka-Fujinka Gakkai Zasshi, 23, 831-840. (Japanese)

18) Nakaya, T. (1973) Studies on acid mucopolysaccharides in the human cervix uteri. Nagoya med. J., 18, 295-319. 
19) Ohkawa, R. (1969) Biochemical study on acidic polysaccharides in the human cervix uteri. Nippon Sanka-Fujinka Gakkai Zasshi, 21, 1309-1318. (Japanese)

20) Stevens, V.C., Dickey, R.P., Vorys, N., Denko, C. \& Ullery, J.C. (1966) Synthesis of chondroitin sulfate by the human cervix during the menstrual cycle. Amer. J. Obstet. Gyne., 95, 959-962.

21) Suzuki, S. (1960) Isolation of novel disaccharides from chondroitin sulfates. J. biol. Chem., 235, 3580-3588.

22) Zachariae, F. \& Thorsøe, H. (1966) Hormonal control of acid mucopolysaccharides in the female genital tract. In: Hormones and Connective Tissue, edited by G. Asboe-Hansen, Munksgaard, Copenhagen. pp. 257-281. 